SAVANNAH RIVER LABORATORY TECHNICAL DIVISION

MEMORANDUM
P. L. ROGGENKAMP

FROM: G. F. O'NEILL BFO

CC: R. Maher, SRP

J. A. Kelley, SRL

J. L. Crandal 1

R. W. Benjamin

L. A. Heinrich

J. E. Hoisington

D. E. Hostetler

I. M. Macafee

F. J. MeCrosson

W. R. McDonelI

TIS Files (2)

October 4, 1982

TO:

\author{
G. F. O'NEILL DFO
}

\title{
ADDITIONAL PU-238 PRODUCTION
}

\section{INTRODUCTION}

Possible DOD demands for up to $1 \mathrm{MW}$ thermal of heat sources could be partially met by increasing SRP Pu-238 production. The Pu-238 would be an alternate or supplement to Sr-90 in these heat sources. There is a growing surplus of Np-237 target material which could be used to produce high-assay Pu-238 (83.57). In addition SRP produces low assay Pu-238 ( $\sim 27 \%)$ that could be extracted instead of being sent to the waste tanks.

\section{SUMMARY}

About $216 \mathrm{~kg}$ or $121 \mathrm{kw}$ thermal of additional high-assay Pu-238 could be produced at SRP through FY1997; it would produce power at an incremental cost of $\sim 1160 /$ watt. About $77 \mathrm{~kg}$ or $44 \mathrm{kw}$ thermal of low assay Pu-238 could also be recovered through FY1997; it would generate power at $\sim \$ 1850 /$ watt. These quantities would supply only a fraction $(\sim 1 / 6)$ of the $1 \mathrm{MW}$ demand projection. Also the costs are considerably higher than the costs for $5 x-90$ fission product heat sources. Sr-90 from SRP and Hanford could supply the total $1 \mathrm{MW}$ thermal requirement ( $150 \mathrm{MCi}$ at $\$ 1$ to $\$ 3$ per $\mathrm{Ci}$ ) at costs in the range of $\$ 150$ to $\$ 450$ per watt. 1

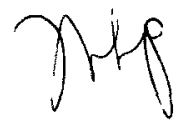




\section{DISCLAIMER}

This report was prepared as an account of work sponsored by an agency of the United States Government. Neither the United States Government nor any agency Thereof, nor any of their employees, makes any warranty, express or implied, or assumes any legal liability or responsibility for the accuracy, completeness, or usefulness of any information, apparatus, product, or process disclosed, or represents that its use would not infringe privately owned rights. Reference herein to any specific commercial product, process, or service by trade name, trademark, manufacturer, or otherwise does not necessarily constitute or imply its endorsement, recommendation, or favoring by the United States Government or any agency thereof. The views and opinions of authors expressed herein do not necessarily state or reflect those of the United States Government or any agency thereof. 


\section{DISCLAIMER}

Portions of this document may be illegible in electronic image products. Images are produced from the best available original document. 


\section{DISCUSSION}

\section{High Assay Pu-238}

The summary tables ${ }^{2}$ in the 1982 Materials Management Report for the Mark 15/Mark 22 operational mode at SRP were used to estimate the surplus Np-237 that would be available each year. This surplus $\mathrm{Np}-237$ was assumed to be irradiated to supply the high-assay Pu-238 for the heat sources.* The data are shown in Table 1.

The incremental cost per kg of extra high assay Pu-238 in FY'83 dollars was estimated as follows:

$\begin{array}{lc}300 \text { Area }^{a} & \$ 4,000 \\ 100 \text { Area } & - \\ 200 \text { Area }^{a} & 110,000 \\ \text { PuFFb } & 211,000 \\ \text { Waste } & 30,000 \\ \text { Equiv. Pu Lostc } & 245,000 \\ \text { Np Burnedd } & \frac{31,000}{\$ 631,000}\end{array}$

a. From Ref. 3 escalated to FY' 83 dollars.

b. Private communication from D. C. Nichols, SRP Separations Dept.

c. Assumes $1.5 \mathrm{~kg}$ of equivalent W.G. Pu lost per $\mathrm{kg}$ of $\mathrm{Pu}-238$ made. The incremental value of W.G. Pu (from the "I" startup) is taken as $\$ 163 / \mathrm{gr}$.

d. Value of $1.4 \mathrm{~kg}$ of $\mathrm{Np}$ burned - incremental recovery cost of surplus Np taken as $\$ 22 / \mathrm{gr}$.

*The 1982 Material Management Plan assumes that the U-236/U-235 ratio in the Mark 22 is maintained at near the current value $(0.14)$. If this ratio is allowed to rise (to $0.6-0.8$ ), so as to limit the SRP requirements for new oralloy from Oak Ridge, the surplus neptunium and thus extra Pu-238 production could increase by a factor of 2 or 3 . 
The low assay Pu-238 production was estimated from the neptunium schedules shown in Table 1. The Mark 22 lattices were assumed to generate the low-assay Pu-238 at an annual rate of $13 \%$ of the Np-237 production. 4 The Mark 16-31 charges in the years prior to FY1985 (before being replaced by Mark 15) were assumed to produce low assay $\mathrm{Pu}-238$ at $9 \%$ of the Np-237 production rate. 4

The cost of low assay Pu-238 recovery through the HB-1ine was developed from an earlier study. 5 Capital costs of $\$ 2.5$ million were required for ion exchange facilities; additional capital. costs of $\$ 1.3$ million were required for $\mathrm{HB}-1$ ine shielding modifications. Operating costs through the canyon and $\mathrm{HB}-\mathrm{line}$ of $\checkmark 300,000$ per $\mathrm{kg}$ were assumed. The current H Area frame capacity may be a pinch point if both the low-assay and high-assay Pu-238 are produced at the same time. In Ref. 3 it was noted that this capacity could be increased by several methods. The PuFF source fabrication costs at $\sim \$ 600,000$ per $\mathrm{kg}$ of $\mathrm{Pu}-238$ were added. These PuFF charges were high compared to the high assay costs because of the large source volume per watt required by the low-assay material.

\section{Production and Costs}

The production and cost values for heat source high-assay Pu-238 are given in Table 2 and for low assay $\mathrm{Pu}-238$ in Table 3 . The costs and production are discounted each year by $10 \%$ and summed to get "Net Present Values". The unit costs are the quotients of the "Net Present Values".

\section{Power Intensity of Pu-238 Sources}

Current high assay $\mathrm{Pu}-238$ sources are fabricated from $\mathrm{PuO}_{2}$ at $85 \%$ of theoretical density with $83.5 \% \mathrm{Pu}-238$ in total plutonium. These sources have a physical density of $9.7 \mathrm{~g} / \mathrm{cc}$ and a power density of 4.0 watt/cc. If low assay Pu-238 sources were fabricated in the same way, at $27 \% \mathrm{Pu}-238$ in the total plutonium, these sources would have a power density of 1.3 watt/cc. Sr-90 sources have a minimum acceptable power density of $\sim 1.0 \mathrm{watt} / \mathrm{cc}$. Therefore, the power density of the Pu-238 sources would be more than adequate. 
P. L. Roggenkamp

4 DPST-82-839

October 4, 1982

Table 1. Np-237 and Pu-238 Schedules at SRP

: Total Np Np Burnup Surplus Np, Extra Pu-238, $\underline{\text { FY }}$ Produced, $\mathrm{kg}$ and Loss, $\mathrm{kg} \mathrm{kg}$

$\mathrm{kg}$

\begin{tabular}{|c|c|c|c|c|}
\hline 1982 & 40.0 & 26.0 & 14.0 & 10.0 \\
\hline 1983 & 42.8 & 10.5 & 32.3 & 23.1 \\
\hline 1984 & 53.2 & 38.8 & 14.4 & 10.3 \\
\hline 1985 & 27.9 & 14.6 & 13.3 & 9.5 \\
\hline 1986 & 27.9 & 13.9 & 14.0 & 10.0 \\
\hline 1987 & 39.2 & 26.3 & 12.9 & 9.2 \\
\hline 1988 & 46.7 & 23.9 & 22.8 & 16.3 \\
\hline 1989 & 50.9 & 25.5 & 25.4 & 18.1 \\
\hline 1990 & 49.2 & 21.3 & 27.9 & 19.9 \\
\hline 1991 & 49.9 & 31.2 & 18.7 & 13.4 \\
\hline 1992 & 50.9 & 27.4 & 23.5 & 16.8 \\
\hline 1993 & 38.3 & 24.8 & 13.5 & 9.6 \\
\hline 1994 & 49.2 & 22.0 & 27.2 & 19.4 \\
\hline 1995 & 44.1 & 27.3 & 16.8 & 12.0 \\
\hline 1996 & 39.7 & 25.6 & 14.1 & 10.1 \\
\hline 1997 & 38.0 & 25.8 & 12.2 & 8.7 \\
\hline 1998 & 37.7 & 30.2 & 7.5 & 5.4 \\
\hline
\end{tabular}


Table 2. High-Assay $\mathrm{Pu}-238$ Production and Costs Discount Rate $=0.1$

\begin{tabular}{|c|c|c|c|c|c|c|c|c|c|c|c|c|c|c|c|c|}
\hline Fiscal Year & 1983 & 1984 & 1985 & 1986 & 1987 & 1988 & 1989 & 1990 & 1991 & 1992 & 1993 & 1994 & 1995 & 1996 & 1997 & Total \\
\hline Oper. Cost,* M\$ & 21.5 & 6.7 & 6.2 & 6.5 & 5.9 & 10.6 & 11.8 & 12.9 & 8.7 & 10.9 & 6.2 & 12.6 & 7.8 & 6.6 & 5.7 & 140.6 \\
\hline Total Cost, M\$ & 21.5 & 6.7 & 11.2 & 21.5 & 15.9 & 10.6 & 11.8 & 12.9 & 8.7 & 10.9 & 6.2 & 12.6 & 7.8 & 6.6 & 5.7 & 140.6 \\
\hline $\begin{array}{l}\mathrm{Pu}-238, \mathrm{kgs} \\
\mathrm{Pu}-238, \mathrm{kw}\end{array}$ & $\begin{array}{l}33.1 \\
18.5\end{array}$ & $\begin{array}{r}10.3 \\
5.7\end{array}$ & $\begin{array}{l}9.5 \\
5.3\end{array}$ & $\begin{array}{r}10.0 \\
5.5\end{array}$ & $\begin{array}{l}9.2 \\
5.1\end{array}$ & $\begin{array}{r}16.3 \\
9.1\end{array}$ & $\begin{array}{l}18.1 \\
10.1\end{array}$ & $\begin{array}{l}19.9 \\
11.1\end{array}$ & $\begin{array}{r}13.4 \\
7.4\end{array}$ & $\begin{array}{r}16.8 \\
9.3\end{array}$ & $\begin{array}{l}9.6 \\
5.3\end{array}$ & $\begin{array}{l}19.4 \\
10.8\end{array}$ & $\begin{array}{r}12.0 \\
6.7\end{array}$ & $\begin{array}{r}10.1 \\
5.6\end{array}$ & $\begin{array}{l}8.7 \\
4.8\end{array}$ & $\begin{array}{l}216.4 \\
121.0\end{array}$ \\
\hline Discount Factor & 1.0 & 0.9090 & 0.8264 & 0.7513 & 0.6830 & 0.6209 & 0.5644 & 0.5131 & 0.4665 & 0.4240 & 0.3855 & 0.3504 & 0.3186 & 0.2896 & 0.2633 & \\
\hline Net Present Values & $s(N P V)$ & & & & & & & & & & & & & & & \\
\hline Oper. NPV & 21.5 & 6.0 & 5.1 & 4.8 & 4.0 & 6.5 & $6.6^{\circ}$ & 6.6 & 4.0 & 4.6 & 2.3 & 4.4 & 2.4 & 1.9 & 1.5 & 82.9 \\
\hline Total NPV & 21.5 & 6.0 & 5.1 & 4.8 & 4.0 & 6.5 & 6.6 & 6.6 & 4.0 & 4.6 & 2.3 & 4.4 & 2.4 & 1.9 & 1.5 & 82.9 \\
\hline NPV of $\mathrm{kg} \mathrm{Pu}-238$ & 33.1 & 9.3 & 7.8 & 7.5 & 6.2 & 10.1 & 10.2 & 10.2 & 6.2 & 7.1 & 3.7 & 6.7 & 3.8 & 2.9 & 2.2 & 127.6 \\
\hline NPV of kw Pu-238 & 18.5 & 5.2 & 4.3 & 4.1 & 3.5 & 5.6 & 5.7 & 5.7 & 3.4 & 3.9 & 2.0 & 3.8 & 2.1 & 1.6 & 1.2 & 71.3 \\
\hline $\begin{array}{ll}\text { Unit } \cos t, & \$ / \mathrm{kg} \\
\text { Unit } \cos t, & \$ / \text { watt }\end{array}$ & & & & & & & & & & & & & & & & $\begin{array}{l}650000 \\
1160.0\end{array}$ \\
\hline
\end{tabular}


Table 3. Low-Assay Pu-238 Recovery and Costs Discount Rate $=0.1$

\begin{tabular}{|c|c|c|c|c|c|c|c|c|c|c|c|c|c|c|c|c|}
\hline Fiscal Year & 1983 & 1984 & 1985 & 1986 & 1987 & 1988 & 1989 & 1990 & 1991 & 1992 & 1993 & 1994 & 1995 & 1996 & 1997 & Total \\
\hline $\begin{array}{l}\text { Capital Cost, } * \text { MS } \\
\text { Oper. Cost, * M\$ }\end{array}$ & $\begin{array}{l}0 \\
5.4\end{array}$ & 6.1 & 3.0 & $\begin{array}{l}0.8 \\
3.0\end{array}$ & $\begin{array}{l}3.0 \\
4.9\end{array}$ & 5.3 & 5.9 & 5.7 & 5.7 & 5.9 & 4.1 & 5.7 & 4.9 & 4.8 & 4.1 & $\begin{array}{r}3.8 \\
74.5\end{array}$ \\
\hline Total Cost, MS & 5.4 & 6.1 & 3.0 & 3.8 & 7.9 & 5.3 & 5.9 & 5.7 & 5.7 & 5.9 & 4.1 & 5.7 & 4.9 & 4.8 & 4.1 & 78.3 \\
\hline $\begin{array}{l}\mathrm{Pu}-238, \mathrm{kgs} \\
\mathrm{Pu}-238, \mathrm{kw}\end{array}$ & $\begin{array}{l}5.1 \\
2.9\end{array}$ & $\begin{array}{l}5.8 \\
3.3\end{array}$ & $\begin{array}{l}2.8 \\
1.6\end{array}$ & $\begin{array}{l}2.8 \\
1.6\end{array}$ & $\begin{array}{l}4.6 \\
2.6\end{array}$ & $\begin{array}{l}5.7 \\
3.2\end{array}$ & $\begin{array}{l}6.4 \\
3.6\end{array}$ & $\begin{array}{l}6.1 \\
3.5\end{array}$ & $\begin{array}{l}6.2 \\
3.5\end{array}$ & $\begin{array}{l}6.4 \\
3.6\end{array}$ & $\begin{array}{l}4.4 \\
2.5\end{array}$ & $\begin{array}{r}6.1 \\
3.5\end{array}$ & $\begin{array}{l}5.3 \\
3.0\end{array}$ & $\begin{array}{l}4.6 \\
2.6\end{array}$ & $\begin{array}{l}4.4 \\
2.5\end{array}$ & $\begin{array}{l}76.7 \\
43.6\end{array}$ \\
\hline
\end{tabular}

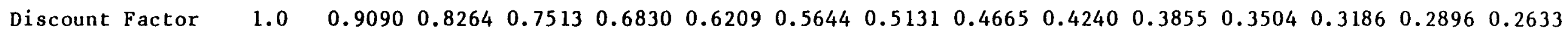

Net Present Values (NPV)

\begin{tabular}{|c|c|c|c|c|c|c|c|c|c|c|c|c|c|c|c|c|}
\hline $\begin{array}{l}\text { Capital NPV } \\
\text { Oper. NPV }\end{array}$ & $\begin{array}{l}0 \\
5.4\end{array}$ & $\begin{array}{l}0 \\
5.5\end{array}$ & $\begin{array}{l}0 \\
2.5\end{array}$ & $\begin{array}{l}0.6 \\
2.3\end{array}$ & $\begin{array}{l}2.0 \\
3.3\end{array}$ & $\begin{array}{l}0 \\
3.3\end{array}$ & $\begin{array}{l}0 \\
3.3\end{array}$ & $\begin{array}{l}0 \\
2.9\end{array}$ & $\begin{array}{l}0 \\
2.6\end{array}$ & $\begin{array}{l}0 \\
2.5\end{array}$ & $\begin{array}{l}0 \\
1.6\end{array}$ & $\begin{array}{l}0 \\
2.0\end{array}$ & $\begin{array}{l}0 \\
1.6\end{array}$ & $\begin{array}{l}0 \\
1.4\end{array}$ & $\begin{array}{l}0 \\
1.1\end{array}$ & $\begin{array}{r}2.7 \\
41.3\end{array}$ \\
\hline Total NPV & 5.4 & 5.5 & 2.5 & 2.9 & 5.3 & 3.3 & 3.3 & 2.9 & 2.6 & 2.5 & 1.6 & 2.0 & 1.6 & 1.4 & 1.1 & $\therefore 4.0$ \\
\hline 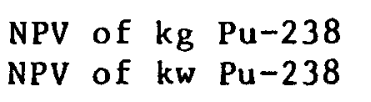 & $\begin{array}{l}5.1 \\
2.9\end{array}$ & $\begin{array}{l}5.3 \\
3.0\end{array}$ & $\begin{array}{l}2.3 \\
1.3\end{array}$ & $\begin{array}{l}2.1 \\
1.2\end{array}$ & $\begin{array}{l}3.1 \\
1.8\end{array}$ & $\begin{array}{l}3.5 \\
2.0\end{array}$ & $\begin{array}{l}3.6 \\
2.0\end{array}$ & $\begin{array}{l}3.1 \\
1.8\end{array}$ & $\begin{array}{l}2.9 \\
1.6\end{array}$ & $\begin{array}{l}2.7 \\
1.5\end{array}$ & $\begin{array}{l}1.7 \\
1.0\end{array}$ & $\begin{array}{l}2.1 \\
1.2\end{array}$ & $\begin{array}{l}1.7 \\
1.0\end{array}$ & $\begin{array}{l}1.3 \\
0.7\end{array}$ & $\begin{array}{l}1.1 \\
0.6\end{array}$ & $\begin{array}{l}41.8 \\
23.8\end{array}$ \\
\hline $\begin{array}{ll}\text { Unit } \operatorname{Cost}, & \$ / \mathrm{kg} \\
\text { Unit } \operatorname{Cost}, & \$ / \text { watt }\end{array}$ & & & & & & & & & & & & & & & & $\begin{array}{l}1050000 \\
1850.0\end{array}$ \\
\hline
\end{tabular}


P. L. Roggenkamp

DPST $-82-830$

October 4,1982

Reserences

1. "Sr-90 and Pm-147 Recovery", J. E. Hoisington and W. R. HoDone11, DPST-82-813, August 30, 1982.

2. "FY-82 SR Materials Management Plan - Summary Report", DPSPWD-82-9-1, August 6, 1982 (Secret-WD).

3. Pu-238 Production Capability, I. M. Macafee and G. F. O'Neill, DPST-80-255, February 14, 1980.

4. "Reactor Dept. - June 1982 Quarterly Forecast Data", DPSP-82-159-2, R. S. Downey, June 27, 1982 (Secret).

5. "Low Assay Pu-238 at SRP", DPST-80-379, G. F. O'Neill, April 23, 1980 (Secret). 